\title{
Vínculo materno-fetal: implicaciones en el desarrollo psicológico y propuesta de intervención en atención temprana
}

\section{Maternal-foetal bonding: implications for psychological development and proposal for early intervention}

\author{
Claudia Patricia Roncallo, Manuel Sánchez de Miguel y Enrique Arranz Freijo \\ Universidad del País Vasco, España
}

Disponible online 31 de agosto de 2015

\begin{abstract}
Diversas investigaciones en el campo de la salud identifican el vínculo materno-fetal como un precedente significativo de la vinculación post-natal entre la madre y su bebé. El vínculo materno-fetal se asocia con aspectos emocionales y cognitivos que permiten recrear al feto como otro ser humano. Este vínculo se expresa, a través de prácticas de salud dirigidas a buscar la protección y el bienestar del feto. Por otra parte se constata que el estado psicológico de la madre influye en el feto, tanto a nivel neurocomportamental y en las alteraciones en el desarrollo que se manifiestan así mismo en el periodo post-natal, afectando significativamente la relación madre-bebé. Por tanto cabe proponer que las prácticas de atención temprana se extiendan al periodo prenatal como estrategia de prevención, repercutiendo favorablemente en la salud materno-infantil. Para ello se plantea un modelo de intervención interdisciplinar, centrado en el vínculo materno-fetal como factor protector del vínculo en el período post-parto y como potenciador de prácticas de parentalidad positiva que fomente un desarrollo psicológico saludable.
\end{abstract}

Palabras Clave: Vínculo Materno-fetal; Atención Temprana; Embarazo; Desarrollo Psicológico.

Health research has identified maternal-foetal bonding as a significant antecedent of post-natal bonding between mother and baby. Maternal-foetal bonding is associated with the emotional and cognitive aspects that allow the foetus to be recreated as another human being. This bond is expressed in health practices aimed at the protection and welfare of the foetus. It has been observed that the psychological state of the mother affects the foetus at the neurobehavioral level and influences the appearance of developmental disorders that also manifest in the post-natal period, thereby significantly affecting the mother-baby relationship. Thus, early intervention practices could be extended to the prenatal period as a prevention strategy to favourably impact the health of the child and mother. To address this issue, we present an interdisciplinary intervention model, which focuses on maternal-foetal bonding as a protective factor for bonding during the postnatal period, and as an enhancer of positive parenting practices that promote healthy psychological development.

Key Words: Maternal-foetal Bonding; Early Intervention; Pregnancy; Psychological Development.

Correspondencia: Claudia Patricia Roncallo Andrade. Departamento de Procesos Básicos y desarrollo. Facultad de Psicología. Universidad del País Vasco UPV/EHU. Departamento de Procesos Psicológicos Básicos y Desarrollo. Avenida Tolosa 7020018 Donostia. Teléfono 943015699. E-mail: croncallo001@ikasle.ehu.eus. E-mail de los co-autores: Manuel Sánchez de Miguel: manu.sanchez@ehu.eus; Enrique Arranz Freijo e.arranzfreijo@ ehu.eus. 


\section{Definición del vínculo materno-fetal}

El vínculo de apego que establece una madre con su hijo, reconocido por su relevancia en el desarrollo psicológico infantil (Bowlby, 1976), ha constituido un terreno fértil donde diversos estudios e intervenciones se han centrado en establecer los factores relevantes en el origen y desarrollo del mismo, así como en las estrategias para su promoción en la primera infancia (Tizón, 2010). Sin embargo, investigaciones provenientes de diferentes ramas de la salud señalan desde hace más de 20 años, que este vínculo no surge en el período posterior al nacimiento sino que tiene su origen en la etapa prenatal.

Los primeros datos acerca de la existencia del llamado vínculo materno-fetal -de carácter unidireccional desde la madre hacia el feto, frente al carácter bidireccional del vínculo de apego- provienen de investigaciones clásicas acerca de la pérdida perinatal y el dolor asociado a la misma. Provienen también del estudio de la relación existente entre la madre y el bebé en el post-parto temprano (Brandon, Pitts, Denton, Stringer y Evans, 2009; Klaus et al., 1972) y del trabajo de Rubin (1967) en el cual, buscando definir la importancia y los factores que intervienen en el inicio del rol materno, postula que la relación existente entre una madre y su hijo recién nacido es consecuencia de un proceso de vinculación prenatal. Sobre esta base, Condon y Dunn (1988) rescatan la hipótesis de Caplan (1961), que afirma que si se conoce la calidad de vinculación de la madre con el feto, es posible predecir la calidad de la vinculación en el post-parto temprano, ya que en la mayoría de los casos ambas vinculaciones son idénticas, constituyendo el parto un episodio de transición entre ellas. (Condon y Dunn, 1988).

Otra fuente de resultados significativos relativos a la construcción teórica del concepto de vínculo materno-fetal, proviene del ámbito empírico de la enfermería. Mecca Cranley (1981) es considerada pionera en establecer tanto una definición operativa (Tabla 1) "el grado en el que las mujeres se dedican a comportamientos que representan una filiación y la interacción con su hijo por nacer" (Cranley, 1981, p.282), como en la creación del primer instrumento de medición del vínculo materno-fetal Maternal fetal attachment scale (Cranley, 1981). Siguiendo esta línea investigadora, Muller (1992) redefine el concepto introduciendo una nueva visión, según la cual el vínculo materno-fetal es una relación única de la madre hacia el feto y es independiente de los sentimientos que ésta tiene acerca de sí misma como madre o como mujer embarazada.

La primera definición procedente del campo de la salud mental, se origina en la investigación acerca del vínculo en el post-parto temprano del psiquiatra australiano John Condon (1988) que plantea que este vínculo es predecible con un alto grado de certeza si se presta atención a la calidad del vínculo de la madre hacia su hijo no nacido. Su teoría de la "primera impresión" abarca tanto aspectos cognitivos, como aspectos afectivos. Dentro de los primeros se encuentran el examen físico minucioso al recién nacido, valorando su comportamiento y apariencia; los segundos se basan en representaciones, que pueden incluir desde un profundo cariño y ternura hasta shock o ambivalencia. La hipótesis central es que el parto no cambia ni instaura el vínculo que las madres sienten hacia su bebé, ya que éste abarca todo el periodo perinatal incluyendo embarazo y post-parto (Condon y Dunn, 1988). Posteriormente Condon y su equipo, definen el vínculo materno-fetal como "el lazo emocional que normalmente se desarrolla entre una mujer embarazada y su hijo no nacido" (Condon y Corkindale, 1997, p.359).

Tabla 1

Definición de vínculo materno-fetal.

\begin{tabular}{cl}
\hline Autor (año) & \multicolumn{1}{c}{ Definición } \\
\hline Cranley (1981) & $\begin{array}{l}\text { El grado en el que las mujeres se dedican a comporta- } \\
\text { mientos que representan una filiación y la interacción } \\
\text { con su hijo por nacer. } \\
\text { Muller (1992) }\end{array}$ \\
$\begin{array}{l}\text { Relación única de la madre hacia el feto y es indepen- } \\
\text { diente de los sentimientos que ésta tiene acerca de sí } \\
\text { misma como madre o como mujer embarazada. }\end{array}$ \\
$\begin{array}{cl}\text { Condon y Corkindale } \\
\text { (1997) }\end{array}$ & $\begin{array}{l}\text { El lazo emocional que normalmente se desarrolla entre } \\
\text { una mujer embarazada y su hijo no nacido. }\end{array}$ \\
\hline
\end{tabular}

Los avances tecnológicos en el campo del diagnóstico prenatal han aumentado el interés de la comunidad científica acerca del vínculo materno-fetal. La posibilidad de acceder a técnicas de ultrasonido que permiten ver más claramente al feto en etapas tempranas de su desarrollo y en tiempo real, han facilitado que los futuros padres imaginen y representen al feto como un ser independiente (Alhusen, 2008; DiPrieto, 2010). Según Brandon et al. (2009) los primeros estudios acerca de cómo la visión temprana del feto en imágenes ecográficas contribuye a la creación de un lazo afectivo con él, datan de la década de los 80. Lumley (1982) sugiere que esta visión permite a las madres pensarlo como una persona real y diferente de sí misma estableciendo una primera relación afectiva.

Si bien existe un consenso entre los investigadores de diferentes disciplinas, acerca de la existencia e importancia del lazo emocional que una mujer establece con su bebé no nacido (Shieh, Kravitz y Wang, 2001), no lo hay en cuanto a la terminología utilizada. Esta controversia en la literatura científica sobre el tema se centra en la utilización del término "apego" para designar esta relación (Condon y Corkindale, 1997; Cranley, 1982; Muller, 1992). Siguiendo las definiciones clásicas acerca del apego (Bowlby, 1976), este se activa en la interacción recíproca madre-bebé, promoviendo la proximidad y contacto necesarios para proporcionar al niño la base segura que le permite la exploración del mundo a la vez que consuelo y protección. El apego es un sistema que se dirige tanto en su componente cognitivo como afectivo hacia el cuidador principal que es esencialmente la madre (Walsh, 2010). Partiendo de esta premisa, algunos autores exponen que las representaciones que activan el lazo emocional de la madre hacia el feto tienen más que ver con un sistema de cuidado, que con uno de apego; debido a que la función de los sistemas de cuidado es la dispo- 
nibilidad y cercanía afectiva de la madre para proveer la atención y protección necesarias para el óptimo desarrollo (Van den Bergh y Simons, 2008).

Otros autores sugieren que el sistema de cuidados concebido como la necesidad y deseo de protección al feto, es parte de una relación más amplia que incluye cognición, emoción y comportamientos y donde se experimentan y expresan afectos de modo estable (Doan y Zimerman, 2003). Siguiendo la línea de estos trabajos (DiPietro, 2010; Doan y Zimerman, 2008), definir el vínculo materno-fetal es una tarea compleja; el mismo, podría ser definido como la filiación de la madre hacia el feto asociada con los aspectos emocionales y cognitivos necesarios para recrearlo como otro ser humano. Como síntesis de los argumentos expuestos hasta ahora, parece oportuno distinguir entre apego como un conjunto de sistemas e interacciones bi-direccionales y el vínculo materno-fetal como un sistema unidireccional con diversas manifestaciones cognitivas, afectivas y comportamentales. Dichas manifestaciones permiten el desarrollo habilidades propias de la función materna, como la protección y la búsqueda de contacto, las cuales se expresan también, en el periodo posterior al nacimiento (Doan y Zimerman, 2003).

En lo referente al estudio de las variables influyentes en el origen y desarrollo del vínculo materno-fetal, autores como Doan y Zimerman (2008) han señalado los cambios en el bienestar psicológico de la madre, diferentes aspectos de la historia personal y múltiples variables psicosociales. De igual manera estos autores indican, que la presencia de habilidades cognitivas y emocionales para recrear al feto como un individuo diferenciado, así como el propio estilo de apego seguro en la infancia o una relación positiva actual con los padres, establecen las bases para un desarrollo positivo del vínculo materno-fetal; dicho vínculo experimenta un incremento exponencial a partir de la percepción de los movimientos fetales y en las últimas semanas de gestación de cara al parto (Damato, 2000; DiPietro, 2010). Dentro de las variables psicosociales se encuentran las características de personalidad de la madre como la empatía, las características específicas del embarazo como la planificación del mismo, el control de la ansiedad y el estrés en los procesos de fertilización asistida, el manejo del impacto emocional de las pérdidas perinatales anteriores, un buen perfil socio-demográfico y un buen apoyo social percibido (Damato, 2000; Doan y Zimerman, 2008).

De la misma forma que las habilidades cognitivas y emocionales son necesarias en el inicio y fortalecimiento del vínculo, su expresión tiene que ver con componentes cognitivos y afectivos. Dentro de los elementos cognitivos se encuentran los pensamientos o representaciones mentales acerca del feto, que permiten a las madres realizar incluso, atribuciones de personalidad y comportamiento; por otra parte, los elementos afectivos hacen referencia a la empatía y el placer en la interacción. Finalmente un último elemento de la expresión del vínculo materno-fetal, se relaciona con comportamientos asociados a las prácticas de salud en función del bienestar del feto, por ejemplo el seguimiento obstétrico, la intención de no hacerle daño y la reducción o finalización del consumo de tabaco, alcohol y otras drogas (Doan y Zimerman, 2008; Yarcheski, Mahon, Yarcheski, Hanks y Cannella, 2009).

Estas prácticas dirigidas a la búsqueda de bienestar en el embarazo y a la protección del óptimo desarrollo fetal constituyen además un fértil campo de estudio; así lo demuestran diversas investigaciones actuales enfocadas en el campo de la salud primal, el cual reúne la vida fetal, parto, post-parto y primer año de vida (Magee et al., 2014; Pisoni, 2014; Shieh, y Kravitz, 2006). Estas investigaciones permiten aumentar el entendimiento y la importancia de la relación materno-fetal, como variable indiscutible en la prevención y en el fortalecimiento y promoción de las prácticas de parentalidad positiva.

\section{Vínculo materno-fetal, estado emocional materno y desarrollo psicológico}

El estudio acerca de la importancia de factores emocionales, ambientales y sociales en el periodo gestacional, ha permitido detallar la influencia del estado psíquico materno tanto en variaciones en tiempo real del comportamiento fetal; como en algunas alteraciones fetales del desarrollo, las cuales presentan un correlato en el periodo post-natal. Así mismo se ha descrito su repercusión en el vínculo materno-fetal y posteriormente en el comportamiento, desarrollo y vinculación neonatal.

Una considerable cantidad de literatura científica ha permitido detallar y conocer entre otras, cómo el grado de ansiedad materna (Allison, Stafford y Anumba, 2011; Talge, Neal y Glover, 2007), el estrés percibido (DiPietro, 2012; HernándezMartínez, Arija, Balaguer, Cavallé y Canals, 2008), la presencia de depresión (Alhusen, Hayat y Gross, 2013; Lindgren, 2001) y los factores socio-económicos poco favorables (Alhusen, Gross, Hayat Rose y Sharps, 2012), se relacionan con el grado de vinculación materno-fetal y con las prácticas de salud o comportamientos que pueden afectar el desarrollo del embarazo y el bienestar fetal. Dentro de estos comportamientos y prácticas de salud se encuentran la abstinencia o reducción del consumo de alcohol (Sedgmen, McMahon, Cairns, Benzie y Woodfield, 2006), del tabaco (Magee, et al., 2014; Massey et al., 2015) y de otras drogas (Shieh y Kravitz, 2006), así como los efectos positivos del descanso, la alimentación adecuada y la atención prenatal (Lindgren, 2001).

Estudios realizados tradicionalmente desde la medicina materno-fetal y la psicobiología han descrito dos tipos de cambios relacionados con la influencia del estado psicológico de la madre durante el embarazo (Tabla 2). Los primeros inducidos por medio de la manipulación experimental de dicho estado, indican variaciones en tiempo real de la dinámica del comportamiento fetal, principalmente aspectos medibles que se expresan así mismo en el recién nacido tales como los patrones de frecuencia cardiaca, su variabilidad o estabilidad y la actividad motora (DiPrieto, 2010, 2012); este comportamiento 
Tabla 2

Influencia del estado psicológico materno en el feto.

\begin{tabular}{cll}
\hline Cambios presentados & \multicolumn{1}{c}{ Origen } & \multicolumn{1}{c}{ Expresión } \\
\hline $\begin{array}{c}\text { Variaciones del } \\
\text { comportamiento fetal }\end{array}$ & $\begin{array}{l}\text { Manipulación experimental del estado psicológico } \\
\text { de la madre durante el embarazo. }\end{array}$ & $\begin{array}{l}\text { Cambio en los patrones de frecuencia cardiaca, su variabilidad o estabilidad y en la } \\
\text { actividad motora. } \\
\text { 1. Hipótesis biológica: Aumento en los niveles de cortisol materno que afectaría al } \\
\text { óptimo desarrollo del eje hipotálamo-pituitario-adrenal fetal. Escasa autorregulación, } \\
\text { déficit en la maduración motora y un menor cociente intelectual infantil. }\end{array}$ \\
$\begin{array}{l}\text { Alteraciones fetales } \\
\text { del desarrollo }\end{array}$ & Ansiedad y estresis psicosocial: Puntuaciones maternas de ansiedad rasgo se caracterizan por la \\
& & $\begin{array}{l}\text { estabilidad pre y post-parto, lo que conduciría a dificultades en la interacción de la madre } \\
\text { con el neonato. Desbordamiento ante la crianza, valoración más negativa del bebé. } \\
\text { 3. Hipótesis biopsicosocial: la expresión en el neurodesarrollo fetal de los efectos de } \\
\text { la exposición prolongada a la ansiedad y el estrés, se ve reforzada por experiencias } \\
\text { ambientales post-natales. Bebés irritables y con menor capacidad de regulación que son } \\
\text { percibidos de forma más negativa, influyendo en las interacciones madre-bebé y en las } \\
\text { pautas de crianza. }\end{array}$ \\
\hline
\end{tabular}

refleja además, los procesos de desarrollo y maduración del sistema nervioso central (Kurjak, Stanojević, Predojević, Laušin y Salihagić-Kadić, 2012). Dentro de las investigaciones en este campo, se encuentran las de exposición materna a la tarea Stroop Color-Word la cual se relaciona con una importante respuesta del sistema nervioso simpático en la madre. Realizadas entre las 24 y 36 semanas de embarazo, han permitido observar un aumento de la frecuencia cardiaca fetal y supresión de la actividad motora con retorno a los niveles basales al finalizar la prueba. Otro ejemplo se encuentra en la visualización controlada por parte de las madres de un documental sobre el parto, donde la respuesta fetal incluía disminución de la actividad motora y del ritmo cardiaco, encontrando una significativa diferencia en los fetos de mujeres primíparas, los cuales mostraron un aumento transitorio de la actividad motora durante la escena de parto propiamente dicha. También la relajación materna ha sido estudiada por medio de audio-visualizaciones guiadas, generando una disminución de la tensión fisiológica de la madre a la vez que disminución de la frecuencia cardiaca fetal (Diprieto, 2012).

El segundo tipo de cambios muestra que la ansiedad materna y el estrés percibido originan alteraciones en el desarrollo fetal que tienen un correlato en el período post-natal (Shieh y Kravitz, 2006); cambios que para Hernández-Martínez et al. (2008) tienen tres posibles explicaciones. La primera, hace referencia a la hipótesis biológica y forma parte de un campo teórico conocido como "programación fetal", el cual describe la adaptación fisiológica del feto al entorno en el cual se desarrolla (Talge et al., 2007). Esta hipótesis expone que el aumento en los niveles de cortisol materno afectaría al óptimo desarrollo del eje hipotálamo-pituitario-adrenal fetal (Christian, 2012), con manifestaciones post-natales como una escasa autorregulación, déficit en la maduración motora y un menor cociente intelectual infantil (Allison et al., 2011, DiPrieto, 2012; HernándezMartínez et al., 2008). Grados altos y sostenidos de ansiedad y estrés pueden además, conducir a un aumento en la liberación de catecolaminas aumentando la resistencia de la arteria uterina y la presión arterial, causando disminución en el flujo sanguíneo uterino y un déficit de oxigenación al feto, los cuales se relacionan con menor perímetro craneal y menor circunferencia abdominal fetal (Allison et al., 2011).
En segundo lugar, la hipótesis psicosocial expone que las puntuaciones de ansiedad rasgo se caracterizan por la estabilidad pre y post-parto lo que conduciría a dificultades en la interacción de la madre con el neonato (Allison et al., 2011; Hernández-Martínez et al., 2008). Datos al respecto apuntan a que mujeres con altas puntuaciones de ansiedad en la gestación, también presentan altas puntuaciones después del parto y muestran tendencia a sentirse más desbordadas ante las tareas propias de la primera crianza haciendo, a su vez, valoraciones más negativas de la conducta o temperamento de sus bebés (DiPrieto, 2012). En último lugar, la hipótesis biopsicosocial plantea que la expresión en el neurodesarrollo fetal de los efectos de la exposición prolongada a la ansiedad y el estrés, se ve reforzada por experiencias ambientales post-natales. Bebés más irritables y con menor capacidad de autorregulación son percibidos de forma más negativa por las madres, quienes pueden presentar alteraciones emocionales pre y post-natales, influyendo de esta forma significativamente en las interacciones y pautas de cuidados y crianza (DiPrieto, 2012; HernándezMartínez et al., 2008).

En cuanto a la depresión prenatal, la literatura indica que al igual que la ansiedad, se caracteriza por su estabilidad en el período post-natal e influye de forma negativa en el crecimiento intrauterino del niño, nacimientos prematuros, la regulación neurocomportamental del neonato y problemas de conducta en la infancia (Goecke et al., 2012). Los síntomas asociados a la depresión durante el embarazo son irritabilidad, tristeza, baja autoestima, sensación de inutilidad y hostilidad hacia el feto (Pollock y Percy, 1999) y según los datos de la investigación realizada por Goecke et al. (2012), la presencia de dichos síntomas durante el tercer trimestre de embarazo se mantiene estable a las tres semanas y seis meses post-parto, afectando significativamente la relación madre-bebé y las prácticas de crianza (Allison et al., 2011; DiPrieto, 2012; Goecke et al., 2012; Lindgren, 2001).

Es preciso anotar que tanto la ansiedad, el estrés percibido y la sintomatología depresiva en una etapa compleja y dinámica como el embarazo, están en gran medida influidos por factores sociodemográficos como la presencia de pareja estable, el nivel de estudios, el nivel socioeconómico y la aceptación social del embarazo, originando una hipótesis multifactorial 
acerca de cómo el estado emocional de la madre influye en el desarrollo del feto y el vínculo materno-fetal (Alhusen et al., 2012). Mujeres expuestas a niveles crónicos de estrés psicosocial tienen una probabilidad más alta de desarrollar angustia o depresión durante el embarazo, comprometiendo de esta forma la vinculación afectiva con el feto y las prácticas de salud asociadas al bienestar del mismo, como la abstinencia en el consumo de alcohol (Sedgmen et al., 2006), tabaco (Magee et al., 2014; Massey et al., 2015), drogas (Shieh y Kravitz, 2006) y el acceso y utilización de recursos de educación y atención prenatal (Lindgren, 2001).

Por otra parte, se ha encontrado una fuerte correspondencia entre los datos obtenidos de las mediciones de ansiedad rasgo, el estrés percibido y la depresión, con la dificultad para generar empatía hacia el feto y reconocerlo como otro diferenciado de sí mismo (Goecke et al., 2012; Pisoni et al., 2014), ambos factores fundamentales para el desarrollo del vínculo materno-fetal (Condon y Corkindale, 1997; Doan y Zimerman, 2008); por lo tanto altos índices de ansiedad, estrés y depresión se asocian con niveles más bajos de vínculo materno-fetal.

La investigación realizada por Massey et al. (2015) respecto al consumo de tabaco, señala como dato relevante que un obstáculo de importancia en la búsqueda de mejores resultados en la abstinencia de tabaco en mujeres embarazadas, radica en una comprensión errónea del porqué las mismas efectúan cambios relacionados con pautas de salud en el embarazo. La posible respuesta es el grado de vinculación afectiva con el feto. En su estudio plantean cómo los procesos conductuales y psíquicos que se ponen de manifiesto al dejar de fumar, difieren esencialmente en mujeres no embarazadas y embarazadas, ya que para estas últimas el deseo de cuidar y proteger al feto actúa como motivación principal para reducir el consumo y en la posterior abstinencia; menor vínculo materno-fetal se asocia con un mayor número de cigarrillos por día durante todo el embarazo. Por otra parte, las mujeres que lograron una reducción considerable del consumo argumentaban el bienestar del bebé como motivación principal, afirmación que denota un comportamiento altruista, empatía y filiación afectiva hacia el feto (Massey et al., 2015). Resultados similares fueron hallados por Sedgmen et al. (2006), quienes observaron una reducción significativa en el número de copas ingeridas en mujeres con problemas de abuso de alcohol, asociadas al incremento del vínculo materno-fetal.

También se constata amplia relación entre los datos proporcionados por investigaciones acerca del consumo de tabaco en mujeres embarazadas y aquellos provenientes de investigaciones acerca del consumo de drogas ilícitas realizados por Shieh y Kravitz (2006). Estos autores sugieren que la posibilidad de iniciar un tratamiento de desintoxicación o acudir a un control gineco-obstétrico o de enfermería en etapas iniciales del embarazo, se asocia con el tipo de droga consumida y con las dimensiones cognitiva y afectiva del vínculo materno-fetal que, como se ha descrito anteriormente, explican la capacidad para representar al feto como otro ser humano, imaginarlo y establecer una filiación afectiva con él (Doan y Zimerman, 2008).

La evidencia empírica plantea que el vínculo maternofetal se relaciona igualmente con la sensibilidad materna, la sincronización de la diada madre-bebé en el post-parto y el establecimiento positivo de la función materna, a la vez que es un precursor del vínculo madre-bebé (Huth-Bocks, Levendosky, Bogat y Von Eye, 2004). Aquellas madres que expresaron sentir una fuerte filiación afectiva hacia sus bebés en el post-parto la describían como un continuo con los sentimientos surgidos hacia el mismo en el periodo prenatal. De la misma forma, mujeres que han desarrollado la empatía y la sensibilidad propias del rol materno en el embarazo, tendían a mayor capacidad para ofrecer una respuesta adecuada y contingente a las necesidades del recién nacido (Siddiqui y Hägglöf, 2000) favoreciendo la interacción positiva entre ambos (Fonagy, Steele y Steele, 1991). Esta interacción ha sido descrita como la base del vínculo madre-bebé que representa un papel fundamental en el desarrollo social, emocional y cognitivo en la infancia temprana (Alhusen et al., 2013; Dubber, Reck, Müller y Gawlik, 2014).

Una vez analizado su origen y desarrollo parece adecuado proponer que la calidad del vínculo materno-fetal constituye un factor protector del vínculo madre-bebé y por tanto, del desarrollo psicológico posterior.

\section{Evaluación del vínculo materno-fetal}

En la medida en que el conocimiento de la vida intrauterina y de los procesos psíquicos que se desarrollan en la mujer durante el embarazo progresa, un número creciente de investigaciones han centrado su interés en la relación materno-fetal como una variable relevante e interesante dentro de la psicología del desarrollo, la psicología de la salud y la psicopatología (Van den Bergh y Simons, 2009), más allá de la obstetricia y la psicobiología. Generalmente, estos estudios han tratado de describir la influencia de la relación materno-fetal en las prácticas saludables durante el embarazo y en la relación posterior madre-bebe (Huth-Bocks et al., 2004).

Las investigaciones de los últimos 20 años en este contexto se han realizado fundamentalmente con tres herramientas de medición (Tabla 3): 1) Maternal-fetal Attachment Scale, MFAS (Cranley, 1981); 2) Maternal-antenatal Attachment Scale, MAAS (Condon, 1993) y, 3) Prenatal Attachment Inventory, PAI (Muller, 1993). Estas escalas han sido de gran ayuda para conocer y describir el vínculo materno-fetal, si bien es cierto, no han estado exentas de críticas, especialmente al momento de definir el constructo teórico del vínculo materno-fetal.

Van den Bergh y Simons (2009) realizaron una revisión de estos tres instrumentos. Estos autores plantean que las medidas son fiables, pero advierten del riesgo de utilizar el término "apego" en el contexto prenatal, ya que no existen evidencias empíricas que avalen una supuesta reciprocidad en esta relación. Es por ello, que subrayan la importancia de atender a las 
actitudes, sentimientos y comportamientos de la madre durante el periodo prenatal y su inclusión en las correspondientes escalas de medidas, toda vez que evidencian el vínculo de la mujer embarazada hacia su hijo no nato. Se trata en definitiva, de observar cómo la madre expresa su atención y compromiso para con el no nato en el marco de la filiación afectiva desarrollada.

\section{Maternal-fetal Attachment Scale (MFAS)}

Esta escala fue desarrollada por Cranley (1981) en el área de enfermería y es el primer instrumento de medición que se diseñó para el estudio del vínculo materno-fetal. En el mismo se describen cinco aspectos relevantes y frecuentes en la manifestación del vínculo materno-fetal: 1) la diferenciación del yo del feto; 2) la entrega de sí mismo; 3) la interacción con el feto; 4) la atribución de características al feto y, 5) la asunción del rol materno.

La versión final de esta escala consta de 24 ítems repartidos en las cinco subescalas citadas anteriormente. El formato de respuesta se presenta en una escala Likert con puntuaciones de 1 (definitivamente no), 2 (no), 3 (duda), 4 (sí) y 5 (definitivamente sí). Este instrumento se utilizó por primera vez en una pequeña muestra de 71 mujeres abarcando el periodo de gestación entre las semanas 35 y 40.

La fiabilidad de esta escala no fue determinada con precisión en los estudios iniciales de Cranley debido al tamaño reducido de la muestra, pero sí en investigaciones posteriores como la de Muller (1993) donde presentaba una muy buena consistencia interna $(\alpha=.92)$. Es de importancia resaltar que desde su creación en los años 80 hasta la actualidad, la MFAS es la escala más utilizada en la medición del vínculo maternofetal. Estudios posteriores (Bloom, 1995; Lindgren, 2001; Shieh y Kravitz, 2006) han revelado igualmente unos buenos indicadores de fiabilidad para la escala total en el rango de .82 a .91, e igualmente para las cinco subescalas, comprendidas en el rango de .52 a .73 .

\section{Prenatal Attachment Inventory (PAI)}

Este instrumento fue creado a comienzos de los años 90. Desarrollado al igual que la MFAS en el contexto de un postgrado en enfermería, el Prenatal Attachment Inventory (PAI), se basa en las críticas realizadas a este primer instrumento de medida. Es por ello que incluye una serie de componentes cognitivos del vínculo materno-fetal que reflejarían los pensamientos y fantasías que tiene la madre acerca del feto, los cuales se encuentran reseñados en la literatura precedente y que revelan la creciente filiación afectiva (Brandon et al., 2009).

Muller (1992) propone además, que estos sentimientos deben diferenciarse de aquellos que tiene la mujer de sí misma como mujer embarazada o de los que provienen de su percepción de sí misma como madre. Este instrumento consta de 21 ítems que se plantean en una escala de frecuencia en formato Likert: "casi nunca" (0).... "casi siempre" (5) y fue aplicado en una muestra de 336 mujeres embarazadas a partir de la semana 20 de gestación. Presenta un coeficiente de fiabilidad alfa que oscila entre 0,81 y 0,93 para el total de la escala. El estudio de Müller (1993) revela que el cuestionario PAI presenta una buena validez convergente con la escala MFAS $(r=.72, p<.01)$. Por otro lado, Damato (2004) encontró un índice de correlación similar $(r=.74, p<.01)$ con la escala MFAS sosteniendo la convergencia propuesta por Muller (1993).

\section{Maternal Antenatal Attachment scale (MAAS)}

La Maternal Antenatal Attachment Scale (MAAS) es el primer instrumento que no proviene del contexto de la enfermería y que se ubica en el ámbito de la salud mental. Fue diseñado por Condon (1993) con el objetivo de solventar la inadecuación de los instrumentos anteriores al momento de diferenciar la actitud de la madre hacia el feto, de la actitud hacia el embarazo o la maternidad en términos generales. Por lo tanto elaboró la escala teniendo como punto de referencia exclusivamente los sentimientos de la mujer hacia el feto (Alhusen, 2008), en términos del amor sentido de manera subjetiva. Desarrollado en una muestra de 112 mujeres tanto primíparas como multíparas, consta de 19 ítems con respuesta en una escala tipo Likert donde 1 representa ausencia de sentimientos y 5 sentimientos muy fuertes. Estos ítems analizan la calidad del vínculo descrita como la experiencia afectiva de la madre y la intensidad, que hace referencia a la cantidad de tiempo invertido en comportamientos o pensamientos que manifiestan filiación con el feto. Así, de la relación entre ambos componentes surgen las que para este autor son las cinco características principales del vínculo materno-fetal: 1) el deseo de conocer al feto; 2) el deseo de estar e interactuar con él; 3) la necesidad de evitar la pérdida o la separación; 4) la necesidad de protegerlo y, 5) identificarse con él y satisfacer sus necesidades (Condon, 1993; Pollock y Percy, 1999). Este instrumento presenta una muy buena fiabilidad $(\alpha=.82)$ para el total de la escala (Condon, 1993; Van den Bergh y Simons, 2009).

Tabla 3

Instrumentos más utilizados en la evaluación del vínculo materno-fetal.

\begin{tabular}{|c|c|c|c|}
\hline Autor (año) & Instrumento & Características & Fiabilidad \\
\hline Cranley (1981) & $\begin{array}{l}\text { Maternal-fetal } \\
\text { Attachment Scale } \\
\text { MFAS }\end{array}$ & $\begin{array}{l}\text { Cinco subescalas que describen aspectos relevantes y frecuentes en la manifestación del } \\
\text { vínculo materno-fetal: } 1 \text {.- la diferenciación del yo del feto, 2.- la entrega de si mismo, } \\
\text { 3.- la interacción con el feto, 4.- la atribución de características al feto y 5.- la asunción } \\
\text { del rol materno. }\end{array}$ & $\begin{array}{l}\text { Fiabilidad para la escala total en el } \\
\text { rango de } .82 \text { a } .91\end{array}$ \\
\hline Condon (1993) & $\begin{array}{l}\text { Maternal-antenatal } \\
\text { Attachment Scale } \\
\text { MAAS }\end{array}$ & Punto de referencia exclusivamente en los sentimientos de la mujer hacia el feto. & $\begin{array}{l}\text { Fiabilidad para el total de la escala } \\
\alpha=.82\end{array}$ \\
\hline Muller (1993) & $\begin{array}{l}\text { Prenatal Attachment } \\
\text { Inventory } \\
\text { PAI }\end{array}$ & $\begin{array}{l}\text { Describe los componentes cognitivos del vínculo materno-fetal que reflejarían los } \\
\text { pensamientos y fantasías que tiene la madre acerca del feto. }\end{array}$ & $\begin{array}{l}\text { Coeficiente de fiabilidad alfa que } \\
\text { oscila entre } 0.81 \text { y } 0.93 \text { para el total } \\
\text { de la escala. }\end{array}$ \\
\hline
\end{tabular}


Es importante señalar que aunque las tres escalas más comúnmente utilizadas poseen buenas propiedades psicométricas, las mismas han sido diseñadas y probadas en muestras de poblaciones claramente sesgadas, ya que las participantes han sido en términos generales mujeres blancas y de clase media. Por tanto, se desconocen los resultados de estos índices al extrapolar dichas escalas a otro tipo de poblaciones; actualmente existen muy pocos estudios sobre vínculo materno-fetal en mujeres en situación de vulnerabilidad económica, psicosocial o de diferentes etnias (Alhusen, 2008; Alhusen et al., 2012; Lindgren, 2001; Shieh y Kravitz, 2002).

\section{Propuesta de intervención temprana}

A la vista de la revisión de literatura científica efectuada cabe proponer que las prácticas de atención temprana, tradicionalmente centradas en el periodo evolutivo 0-6 años pudieran extenderse al periodo prenatal. En este sentido, el análisis del vínculo materno-fetal en la promoción de la salud maternoinfantil tiene significativas repercusiones teóricas y clínicas. Las teóricas permiten aceptar la complejidad del vínculo existente entre una mujer y su bebé no nato, observando que el vínculo descrito en el periodo neonatal puede asumirse como un continuo del existente en el embarazo, derivando en prácticas de salud fundamentales para el desarrollo del feto.

En lo que se refiere a las implicaciones clínicas, proporcionan una nueva mirada a la intervención en el periodo prenatal especialmente en el campo de la prevención. Acciones dirigidas a mejorar la empatía de las mujeres hacia el feto y el vínculo materno-fetal, pueden optimizar a corto y largo plazo la salud de las madres y sus bebés (Van den Bergh y Simons, 2009), el óptimo desarrollo del vínculo madre bebé (Shieh y Kravitz, 2002, 2006; Siddiqui y Hägglöf, 2000) y las prácticas de crianza y parentalidad positiva.

Los resultados de diversas investigaciones acerca del vínculo materno-fetal alumbran la importancia de conocer los factores que se ponen de manifiesto en la filiación afectiva de la madre hacia su hijo no nato. Esta perspectiva permite la planificación y adecuación de intervenciones en el periodo prenatal, con el fin de cuidar el desarrollo físico del feto y la vinculación posterior del bebé con su madre (Pisoni et al., 2014; Walsh, Hepper, Bagge, Wadephul y Jomeen, 2013). Una intervención de estas características, debe ser concebida necesariamente desde un enfoque interdisciplinar en donde la evaluación y detección de factores de riesgo psicosociales contribuya a mejorar la salud materno-fetal, funcionando a su vez como red de apoyo para la madre.

Los datos acerca de la exposición fetal a elementos teratógenos tales como la ansiedad, el estrés percibido y la depresión descritos en el apartado anterior, apoyan una hipótesis multifactorial sobre cómo los mismos podrían intervenir en el desarrollo del feto y la relación materno-fetal. En consecuencia se plantea una intervención cuyo objetivo último es la promoción de la salud materno-fetal, a través del fortalecimiento de la madre en sus recursos psicológicos y sociales y en el nuevo rol materno por medio de un componente motivacional (el vínculo materno-fetal) y un componente instrumental (las prácticas de salud). Este tipo de intervención trabajaría desde la experiencia interdisciplinar en seis áreas:

a) Evaluación temprana del vínculo materno-fetal. Con varios fines el primero, proteger y potenciar la relación materno-fetal como eje motivacional de la intervención; el segundo, la detección de dificultades en la vinculación y el tercero, la implementación de estrategias individualizadas de atención ante la presencia de asociaciones significativas entre bajos niveles de vínculo materno-fetal y posibles trastornos emocionales gestacionales.

b) Detección temprana de altos niveles de estrés percibido, sintomatología ansiosa o depresiva, con el fin de ofrecer a las mujeres un servicio de psicoterapia especializada en el período perinatal que permita paliar sus efectos nocivos en la relación materno-fetal y en el desarrollo del feto (Goecke et al., 2012). Merece la pena resaltar la importancia de este punto en relación a la consistente asociación entre la depresión durante el embarazo y la depresión post-parto. Si bien son de frecuente aparición los episodios de llanto, los cambios en los patrones de alimentación y sueño y la irritabilidad en el post-parto temprano dando lugar a una alteración leve del estado de ánimo conocida como baby blues, ésta remite en el transcurso de las primeras semanas del puerperio; en contraste, la depresión post-parto como cuadro clínico, tiene una mayor duración, se caracteriza por un estado anímico depresivo grave y requiere tratamiento (Goecke et al. 2012). En consecuencia conocer la presencia de depresión en el embarazo permite actuar preventivamente hacia los efectos de la misma en el post-parto.

c) Construcción de la historia vincular de la madre. La literatura indica que gran parte de la capacidad para desarrollar una identidad materna positiva radica en el propio estilo de apego infantil de la madre, desde el cual se han construido las relaciones con otros y la capacidad de percibirse a sí misma como proveedora de protección y cuidados; ambas cuestiones fundamentales en el establecimiento del vínculo materno-fetal (Shieh y Kravitz, 2006). Los datos demuestran también que mujeres con estilos de apego inseguros que dificultan su vinculación con el feto, no tienden a beneficiarse de intervenciones destinadas a fortalecer la filiación afectiva con sus hijos, ya que la confianza en el otro y la alianza terapéutica pueden estar comprometidas (Alhusen et al., 2013). Conocer la historia vincular de la madre permite re-elaborarla y fortalecer las relaciones afectivas presentes con la pareja, grupo de amigos y familia, siendo estas indispensables para la percepción positiva de apoyo y seguridad durante el embarazo, actuando como fuente de salud.

d) Fortalecimiento y promoción del vínculo materno-fetal. La consulta de ginecología o de matrona así como los espacios de preparación al parto, son una importante oportunidad para el fortalecimiento y la promoción del vínculo materno-fetal. Shieh y Kravitz (2006) exponen que invitar a la madre a ser 
consciente de los movimientos fetales y hablar sobre ellos, repercute de forma positiva en su interés hacia su hijo; de la misma forma se ha observado un incremento en el vínculo asociado con la visión del feto por medio de ultrasonido (Sedgmen et al., 2006). Una atención gineco-obstétrica de calidad, que permita la exploración de otras áreas relevantes en el embarazo además de la fisiológica incorporando y prestando la importancia necesaria al componente del vínculo, puede resultar de gran ayuda en el incremento de la experiencia de afecto hacia el feto y sus correlatos en la salud materno-fetal.

De la misma forma, merece la pena resaltar la importancia de la figura de la pareja de crianza como elemento fundamental en el desarrollo positivo del vínculo materno-fetal. Los datos señalan que la presencia de una pareja estable actúa fortaleciendo a la madre, incidiendo en la percepción del apoyo y afecto necesarios en una etapa cambiante y compleja como es el embarazo (Persson, Fridlund, Kvist y Dykes, 2012). Por otra parte un nuevo campo de investigación actualmente en desarrollo, expone que los padres pueden tener su propio proceso de vinculación afectiva hacia el feto (Condon, 1985, 2006).

e) Promoción de prácticas de cuidado y salud. Las prácticas de salud en el embarazo permiten el adecuado desarrollo del feto así como disminuir los factores de riesgo (Lindgren, 2001). Un enfoque de intervención dirigido a la promoción del vínculo materno-fetal debe a su vez fomentar las prácticas de cuidado y salud en el embarazo a través de la empatía y el deseo de cuidar y proteger al feto, característicos de este vínculo (Shieh y Kravitz, 2006). Es posible establecer una hipótesis según la cual las prácticas de salud actúen como refuerzo positivo del autoconcepto y de la función materna en mujeres en situación de vulnerabilidad y riesgo, permitiendo a su vez un incremento en el vínculo materno-fetal.

f) Creación de nuevas redes sociales y espacios de apoyo. Como punto final, es preciso tener en cuenta la repercusión en la salud física y mental del apoyo social percibido durante el embarazo. Los datos indican que las madres se benefician de los grupos de apoyo madre a madre y de los espacios de encuentro que permiten la expresión de los sentimientos, vivencias y temores propios de esta etapa; como resultado, estos grupos adquieren una importancia significativa en la transición a la maternidad y en el periodo post-parto. Experiencias de este tipo de grupos señalan que los mismos se establecen como redes de apoyo e influyen significativamente en la vinculación positiva con el feto y como algunos estudios lo demuestran, también en el apego infantil (Huth-Bocks et al., 2004). Se han descrito tres factores que surgen como explicación a los beneficios psicosociales de los grupos madre-madre. El primero se encuentra en relación a la exposición a modelos positivos de cuidados de otras madres durante el embarazo o con sus hijos; el segundo, establece que experiencias relacionales positivas mejoran la sensibilidad materna y la capacidad de vinculación afectiva; y el tercero, que el grupo de madres influye significativamente en la percepción de sí mismo y de las relaciones con los otros, hecho que beneficiaría también el vínculo materno-fetal (HuthBocks et al., 2004).

Programas similares de intervención han sido llevados a cabo con excelentes resultados, tal es el caso del Nurse family partnership. Este programa llevado a cabo a través de visitas domiciliarias de enfermeras desde el periodo prenatal hasta los dos años del bebé, busca cambiar las condiciones de las madres en alto riesgo psicosocial a través de la ayuda en el establecimiento de prácticas de salud, las pautas educativas respecto al cuidado y crianza infantil y la mejora de las condiciones económicas de la familia (Alhusen et al., 2013; Segal, Sara Opie y Dalziel, 2012). Una experiencia similar es llevada a cabo por medio del programa Minding the Baby, cuyo principal objetivo es el fortalecimiento del vínculo madre-bebé comenzando en el embarazo. Esta intervención dirigida igualmente a población de riesgo y vulnerabilidad psicosocial (madres adolescentes con historia de trauma) trabaja desde un enfoque interdisciplinar (enfermera y trabajador social), por medio de la psicoterapia basada en la mentalización buscando una mejoría en la capacidad reflexiva de las madres (Alhusen et al., 2013; Slade et al., 2005).

Finalmente, cabe afirmar que una intervención interdisciplinar basada en el vínculo materno-fetal ofrecería efectos positivos a corto y largo plazo de los que las familias pudieran beneficiarse. Potenciar los recursos internos de las mujeres, así como su capacidad de resiliencia y el papel de la pareja de crianza y las redes sociales de apoyo, incide en la salud física y emocional de madres y bebés, pero también en la vinculación post-natal, el apego infantil, las pautas de crianza y parentalidad positiva y la prevención entre otras del abuso y maltrato.

\section{Referencias}

1. Alhusen, J. L. (2008). A literature update on Maternal-Fetal attachment. Journal of Obstetric, Gynecologic, \& Neonatal Nursing, 37, 315-328. http://dx.doi.org/10.1111/j.1552$\underline{6909.2008 .00241 . x}$

2. Alhusen, J. L., Gross, D., Hayat, M. J., Rose, L. y Sharps, P. (2012). The role of mental health on Maternal-Fetal attachment in Low-Income women. Journal of Obstetric, Gynecologic, \& Neonatal Nursing, 41, E71-E81. http://dx.doi.org/10.1111/j.1552-6909.2012.01385.x.

3. Alhusen, J. L., Hayat, M. J. y Gross, D. (2013). A longitudinal study of maternal attachment and infant developmental outcomes. Archives of Women's Mental Health, 16, 521529. http://dx.doi.org/10.1007/s00737-013-0357-8

4. Allison, S. J., Stafford, J. y Anumba, D. O. (2011). The effect of stress and anxiety associated with maternal prenatal diagnosis on feto-maternal attachment. BMC Women's Health, 11, 33. http://dx.doi.org/10.1186/1472-6874-11-33

5. Bloom, K. C. (1995). The development of attachment behaviors in pregnant adolescents. Nursing Research, 44, 284-289. http://dx.doi.org/10.1097/00006199-199509000$\underline{00005}$ 
6. Bowlby, J. (1976). La separación afectiva. Buenos Aires. Paidós.

7. Brandon, A. R., Pitts, S., Denton, W. H., Stringer, C. A. y Evans, H. M. (2009). A history of the theory of prenatal attachment. Journal of Prenatal \& Perinatal Psychology \& Health: APPPAH, 23, 201-222.

8. Christian, L. M. (2012). Psychoneuroimmunology in pregnancy: Immune pathways linking stress with maternal health, adverse birth outcomes, and fetal development. Neuroscience \& Biobehavioral Reviews, 36, 350-361. http://dx.doi.org/10.1016/j.neubiorev.2011.07.005

9. Condon, J. T. (1985). The parental-foetal relationship-a comparison of male and female expectant parents. Journal of Psychosomatic Obstetrics \& Gynecology, 4, 271-284. http://dx.doi.org/10.3109/01674828509016729

10. Condon, J. T. (1993). The assessment of antenatal emotional attachment: Development of a questionnaire instrument. British Journal of Medical Psychology, 66, 167-183. http://dx.doi.org/10.1111/j.2044-8341.1993.tb01739.x

11. Condon, J. T. (2006). What about dad? psychosocial and mental health issues for new fathers. Australian Family Physician, 35, 690.

12. Condon, J. T. y Corkindale, C. (1997). The correlates of antenatal attachment in pregnant women. British Journal of Medical Psychology, 70, 359-372. http://dx.doi.org/10.1111/j.2044-8341.1997.tb01912.x

13. Condon, J. T. y Dunn, D. J. (1988). Nature and determinants of parent-to-infant attachment in the early postnatal period. Journal of the American Academy of Child \& Adolescent Psychiatry, 27, 293-299. http://dx.doi. org/10.1097/00004583-198805000-00005

14. Cranley, M. S. (1981). Development of a tool for the measurement of maternal attachment during pregnancy. Nursing Research, 30, 281-284. http://dx.doi.org/10.1097/00006199-198109000-00008

15. Damato, E. G. (2000). Maternal-Fetal attachment in twin pregnancies. Journal of Obstetric, Gynecologic \& Neonatal Nursing, 29, 598-605. http://dx.doi.org/10.1111/j.1552-6909.2000.tb02073.x

16. Damato, E.G. (2004). Predictors of prenatal attachment in mothers of twins. Journal of Obstetric, Gynecologic and Neonatal Care, 33, 436-445. http://dx.doi.org/10.1177/0884217504266894

17. DiPietro, J. A. (2010). Psychological and psychophysiological considerations regarding the maternal-fetal relationship. Infant and Child Development, 19, 27-38. http://dx.doi.org/10.1002/icd.651

18. DiPietro. J.A. (2012). Maternal stress in pregnancy: Considerations for fetal development. Journal of Adolescent Health, 51, S3-S8. http://dx.doi.org/10.1016/j.jadohealth.2012.04.008

19. Dipietro, J. A., Irizarry, R. A., Costigan, K. A. y Gurewitsch, E. D. (2004). The psychophysiology of the mater- nal-fetal relationship. Psychophysiology, 41, 510-520. http://dx.doi.org/10.1111/j.1469-8986.2004.00187.x

20. Doan, H. M. y Zimerman, A. (2003). Conceptualizing prenatal attachment: Toward a multidimensional view. Journal of Prenatal and Perinatal Psychology and Health, 18, 109.

21. Doan, H. M., y Zimerman, A. (2008). Prenatal attachment: A developmental model. International Journal Prenatal and Perinatal Psychology and Medicine, 20, 20-28.

22. Dubber, S., Reck, C., Müller, M. y Gawlik, S. (2014). Postpartum bonding: The role of perinatal depression, anxiety and maternal-fetal bonding during pregnancy. Archives of Women's Mental Health, 1-9. http://dx.doi.org/10.1007/ s00737-014-0445-4

23. Fenwick, J., Gamble, J., Creedy, D. K., Buist, A., Turkstra, E., Sneddon, A. y Toohill, J. (2013). Study protocol for reducing childbirth fear: A midwife-led psycho-education intervention. BMC Pregnancy and Childbirth, 13, 190. doi:10.1186/1471-2393-13-190. http://dx.doi. org/10.1186/1471-2393-13-190

24. Fonagy, P., Steele, H. y Steele, M. (1991). Maternal representations of attachment during pregnancy predict the organization of infant-mother attachment at one year of age. Child Development, 62, 891-905. http://dx.doi. org/10.2307/1131141

25. Goecke, T., Voigt, F., Faschingbauer, F., Spangler, G., Beckmann, M. y Beetz, A. (2012). The association of prenatal attachment and perinatal factors with pre-and postpartum depression in first-time mothers. Archives of Gynecology and Obstetrics, 286, 309-316. http://dx.doi.org/10.1007/ s00404-012-2286-6

26. Hernández-Martínez, C., Arija, V., Balaguer, A., Cavallé, P. y Canals, J. (2008). Do the emotional states of pregnant women affect neonatal behaviour? Early Human Development, 84, 745-750. http://dx.doi.org/10.1016/j.earlhumdev.2008.05.002

27. Huth-Bocks, A. C., Levendosky, A. A., Bogat, G. A. y Von Eye, A. (2004). The impact of maternal characteristics and contextual variables on infant-mother attachment. Child Development, 75, 480-496. http://dx.doi.org/10.1111/ j.1467-8624.2004.00688.x

28. Klaus, M. H., Jerauld, R., Kreger, N. C., McAlpine, W., Steffa, M. y Kennel, J. H. (1972). Maternal attachment. importance of the first post-partum days. The New England Journal of Medicine, 286, 460-463. http://dx.doi. org/10.1056/NEJM197203022860904

29. Kurjak, A., Stanojević, M., Predojević, M., Laušin, I. y Salihagić-Kadić, A. (2012). Neurobehavior in fetal life. Seminars in Fetal and Neonatal Medicine, 17, 319-323. http://dx.doi.org/10.1016/j.siny.2012.06.005

30. Lindgren, K. (2001). Relationships among maternal-fetal attachment, prenatal depression, and health practices in pregnancy. Research in Nursing \& Health, 24, 203-217. http://dx.doi.org/10.1002/nur.1023 
31. Magee, S. R., Bublitz, M. H., Orazine, C., Brush, B., Salisbury, A., Niaura, R. y Stroud, L. R. (2014). The relationship between Maternal-Fetal attachment and cigarette smoking over pregnancy. Maternal and Child Health Journal, 18, 1017-1022. http://dx.doi.org/10.1007/s10995-013-1330-x

32. Massey, S. H., Bublitz, M. H., Magee, S. R., Salisbury, A., Niaura, R. S., Wakschlag, L. S. y Stroud, L. R. (2015). Maternal-fetal attachment differentiates patterns of prenatal smoking and exposure. Addictive Behaviors, 45, 51-56. http://dx.doi.org/10.1016/j.addbeh.2015.01.028

33. Muller, M. E. (1992). A critical review of prenatal attachment research. Research and Theory for Nursing Practice, 6, 5-22.

34. Muller, M. E. (1993). Development of the prenatal attachment inventory. Western Journal of Nursing Research, 15, 199-215. http://dx.doi.org/10.1177/019394599301500205

35. Persson, E. K., Fridlund, B., Kvist, L. J. y Dykes, A. (2012). Fathers' sense of security during the first postnatal week-A qualitative interview study in Sweden. Midwifery, 28, e697e704. http://dx.doi.org/10.1016/j.midw.2011.08.010

36. Pisoni, C., Garofoli, F., Tzialla, C., Orcesi, S., Spinillo, A., Politi, P. y Stronati, M. (2014). Risk and protective factors in maternal-fetal attachment development. Early Human Development, 90, S45-S46. http://dx.doi.org/10.1016/ $\underline{\mathrm{S} 0378-3782(14) 50012-6}$

37. Pollock, P. H. y Percy, A. (1999). Maternal antenatal attachment style and potential fetal abuse. Child Abuse \& Neglect, 23, 1345-1357. http://dx.doi.org/10.1016/S0145-2134(99)00101-5

38. Rubin, R. (1967). Attainment of the maternal role: part I. processes. Nursing Research, 16, 237-245. http://dx.doi. org/10.1097/00006199-196701630-00006

39. Sedgmen, B., McMahon, C., Cairns, D., Benzie, R. y Woodfield, R. (2006). The impact of two-dimensional versus three-dimensional ultrasound exposure on maternal-fetal attachment and maternal health behavior in pregnancy. Ultrasound in Obstetrics \& Gynecology, 27, 245-251. http://dx.doi.org/10.1002/uog.2703

40. Segal, L., Sara Opie, R. y Dalziel, K. (2012). Theory! the missing link in understanding the performance of Neonate/Infant Home-Visiting programs to prevent child maltreatment: A systematic review. Milbank Quarterly, 90, 47-106. http://dx.doi.org/10.1111/j.1468-0009.2011.00655.x

41. Shieh, C. y Kravitz, M. (2002). Maternal-Fetal attachment in pregnant women who use illicit drugs. Journal of Obstetric, Gynecologic, \& Neonatal Nursing, 31, 156-164. http://dx.doi.org/10.1177/088421702129004778

42. Shieh, C. y Kravitz, M. (2006). Severity of drug use, initiation of prenatal care, and Maternal-Fetal attachment in pregnant marijuana and Cocaine/Heroin users. Journal of Obstetric, Gynecologic \& Neonatal Nursing, 35, 499-508. http://dx.doi.org/10.1111/j.1552-6909.2006.00063.x
43. Shieh, C., Kravitz, M. y Wang, H. H. (2001). What do we know about maternal fetal attachment? The Kaohsiung Journal of Medical Sciences, 17, 448-454.

44. Siddiqui, A. y Hägglöf, B. (2000). Does maternal prenatal attachment predict postnatal mother-infant interaction? Early Human Development, 59, 13-25. http://dx.doi.org/10.1016/S0378-3782(00)00076-1

45. Slade, A., Sadler, L., Dios-Kenn, C., Webb, D., CurrierEzepchick, J. y Mayes, L. (2005). Minding the baby: A reflective parenting program. Psychoanalytic Study of the Child, 60, 74-100.

46. Talge, N. M., Neal, C. y Glover, V. (2007). Antenatal maternal stress and long-term effects on child neurodevelopment: How and why? Journal of Child Psychology and Psychiatry, 48, 245-261. http://dx.doi.org/10.1111/j.14697610.2006.01714.x

47. Tizón, J. L. (2010, Octubre). Funciones psicosociales de la familia y cuidados tempranos en la infancia. Crisis económica y políticas sociales. Ponencia presentada en el II Congreso Anual De La Red Española De Políticas Sociales, Madrid.

48. Van den Bergh, B., Mulder, E., Mennes, M. y Glover, V. (2005). Antenatal maternal anxiety and stress and the neurobehavioural development of the fetus and child: links and possible mechanisms. A review. Neuroscience \& Biobehavioral Reviews, 9, 237-58. http://dx.doi.org/10.1016/j.neubiorev.2004.10.007

49. Van den Bergh, B. y Simons, A. (2009). A review of scales to measure the mother-fetus relationship. Journal of Reproductive and Infant Psychology, 27, 114-126. http://dx.doi. org/10.1080/02646830802007480

50. Walsh, J. (2010). Definitions matter: If maternalfetal relationships are not attachment, what are they? Archives of Women's Mental Health, 13, 449-451. http://dx.doi.org/10.1007/s00737-010-0152-8

51. Walsh, J., Hepper, E. G., Bagge, S. R., Wadephul, F. y Jomeen, J. (2013). Maternal-fetal relationships and psychological health: Emerging research directions. Journal of Reproductive and Infant Psychology, 31, 490-499. http://dx.doi.org/10.1080/02646838.2013.834311

52. Yarcheski, A., Mahon, N. E., Yarcheski, T. J., Hanks, M. M. y Cannella, B. L. (2009). A meta-analytic study of predictors of maternal-fetal attachment. International Journal of Nursing Studies, 46, 708-715. http://dx.doi.org/10.1016/j. ijnurstu.2008.10.013

Fecha de recepción: 26 de febrero, 2015 Fecha de recepción de la versión modificada: 4 de mayo, 2015 Fecha de aceptación: 7 de junio, 2015 